Pedagogía y Saberes No. 47

Universidad Pedagógica Nacional

Facultad de Educación. 2017, pp. 79-93

\title{
El discurso de las competencias y la domesticación de la crítica en la educación superior* $^{*}$
}

Artículo de investigación

The Discourse of Competences and the Domestication of Critical Analysis in Higher Education

O discurso das competências e a domesticação da crítica na educação superior

Juan Pablo Sánchez Rojas**

Para citar este artículo:

Sánchez, J. (2017). El discurso de las competencias y la domesticación de la crítica en la educación superior. Pedagogía y Saberes (47), 79-93.

* Artículo de reflexión resultado de la investigación "Las competencias comunicativas en documentos públicos e institucionales de educación superior colombiana, 2003-2013. Una mirada desde el análisis crítico del discurso" (código: PCI-2014-61), aprobado y financiado por la Vicerrectoría de Investigaciones de la Universidad El Bosque, a través de la "VI Convocatoria interna para la financiación de proyectos de investigación e innovación tecnológica-2014".

** Profesor Asistente del Departamento de Humanidades de la Universidad El Bosque. Magister en filosofía de la Pontificia Universidad Javeriana. Correo: jpsanchez1001@hotmail.com

Perfil ORCID: http:// orcid.org/0000-0002-1795-958X 


\title{
Resumen
}

Este artículo aborda algunas de las conclusiones obtenidas de un análisis crítico sobre el discurso de las competencias en la educación superior colombiana, a partir de una mirada foucaultiana a las relaciones y modos de ejercicio del poder en documentos de política pública producidos principalmente por el Ministerio de Educación Nacional. Entre los resultados del análisis se destaca en primer lugar, la problematización del discurso de las competencias que se presente como favorable a la formación de sujetos críticos y autónomos y, en segundo lugar, el análisis de las implicaciones que trae el hecho de que este discurso opere a través de técnicas disciplinarias, que pretenden encauzar las conductas críticas de la población y, así, conjurar sus poderes y peligros.

\section{Palabras clave}

Foucault; crítica; poder disciplinario; sociedades de control; competencias; educación superior colombiana

\begin{abstract}
This paper discusses some of the conclusions obtained from a critical analysis of the discourse of competencies in Colombian higher education, from a Foucauldian perspective, the paper addresses the relations and modes of exercising power in public policy documents produced mainly by the Colombian Ministry Education. Among the results of the analysis, special attention is drawn to the problematization of the discourse of the competencies, presented as favorable to shaping critical and autonomous individuals and, secondly, to the analysis of the implications of the fact that this discourse operates through disciplinary techniques, which seek to control the critical behavior of the population and, thus, invoke their powers and dangers.
\end{abstract}

\section{Keywords}

Foucault; criticism, disciplinary power; societies of control; competencies; Colombian higher education

\section{Resumo}

Neste artigo são abordadas algumas das conclusões obtidas de uma análise crítica sobre o discurso das competências na educação superior colombiana, desde um olhar foucaultiano sobre as relações e formas de exercício do poder em documentos de política pública produzidos principalmente pelo Ministério da Educação Nacional. Entre os resultados da análise, assinala-se, em primeiro lugar, a problematização do discurso das competências, apresentado como elemento favorável à formação de sujeitos críticos e autônomos. Em segundo lugar, analisam-se as implicações que esse discurso opere através de técnicas disciplinarias que visam canalizar as condutas críticas da população e, assim, conjurar seus poderes e perigos.

\section{Palavras chave}

Foucault; crítica; poder disciplinar; sociedades de controle; competências; educação superior colombiana 


\section{Introducción}

$\mathrm{E}$ n los últimos años, el lenguaje de las competencias se ha tornado hegemónico en las prácticas discursivas del Estado, las instituciones y algunos docentes e investigadores de la educación superior colombiana. Lejos de ser simplemente un discurso, este lenguaje ha invadido las prácticas educativas en las universidades, de tal modo que actualmente los reglamentos, las políticas, los perfiles profesionales, los currículos, las estrategias pedagógicas y las prácticas concretas de formación y evaluación se han articulado a este discurso. En este artículo abordaré algunas de las conclusiones obtenidas en un análisis crítico previo de documentos que le han permitido al Estado direccionar las prácticas pedagógicas en las universidades hacia la formación y evaluación por competencias ${ }^{1}$. En el análisis aposté por una perspectiva crítica desde algunos planteamientos filosóficos de Michel Foucault, con miras a descifrar relaciones y modos de ejercicio del poder en las prácticas discursivas analizadas. En este texto reflexionaré en torno a dos conclusiones centrales del análisis, profundizando algunos conceptos y problematizaciones desde esta mirada filosófica. En el primer apartado, analizaré el concepto de crítica y su relación con la noción de Ilustración en Foucault, con el fin de volver a la ruta metodológica trazada en la investigación y mostrar algunos de sus alcances políticos. Con base en este concepto, en el segundo apartado problematizaré el hecho de que el discurso de las competencias en la educación superior colombiana se asimile a un discurso moderno y crítico, favorable a la formación de sujetos que se gobiernen a sí mismos. En el tercer apartado, expondré cómo este discurso se articula en un dispositivo de normalización de las conductas críticas, a través de la introducción de un procedimiento típicamente disciplinario: el examen (de Estado). Con este recorrido espero mostrar cómo el discurso de las competencias en la educación superior colombiana intenta disociar el uso económico del gobierno de sí de posibles usos políticos que puedan amenazar las actuales estrategias de gobierno de la población.

1 Los resultados de este análisis fueron expuestos en la ponencia "Estandarización de competencias y formación de sujetos flexibles en las sociedades de control. Un análisis crítico del discurso desde una perspectiva foucaultiana", presentada en el XI Seminario Internacional de la Red Estrado, "Movimientos pedagógicos y trabajo docente en tiempos de estandarización", celebrado en México D.F., los días 16, 17 y 18 de noviembre de 2016. Esta ponencia ha sido publicada en los anales del Seminario: $\mathrm{http}: / /$ redeestrado.org/xi_seminario/.

\section{La crítica en Foucault}

Me gustaría empezar por retomar el concepto de crítica de Michel Foucault, por dos razones: la primera, porque la perspectiva asumida tanto en el análisis discursivo realizado como en este artículo parte de los abordajes que hizo Foucault a dicho concepto, entre finales de los setenta y mediados de los ochenta. Esto significa que he tomado del autor francés, además de algunos de sus conceptos, una interpretación de su forma de mirar, de su manera de abordar y problematizar los asuntos de los que se ocupó, que podemos encontrar en algunos de sus trabajos, sobre todo genealógicos, de mediados de los años setenta (Foucault, 2009, 2011b). Volver sobre esta noción me permitirá, pues, revisar algunas implicaciones de la apuesta por un análisis crítico desde una mirada foucaultiana. Cabe aclarar que en este artículo no expondré el enfoque metodológico y los procedimientos específicos del análisis ya realizado, pues esto desbordaría sus propósitos de reflexión, de vuelta sobre las conclusiones de dicho análisis. La segunda razón es que la noción de crítica y su relación con la Ilustración son claves para las consideraciones de los siguientes apartados, en los que se confrontan estas nociones con el modo en que se usan y se articulan en el contexto discursivo local analizado. De este modo, resultan necesarias en el desarrollo del argumento central del artículo.

A finales de los años 1970 e inicios de 1980, Foucault se dedicó al análisis de la gubernamentalidad, desde la perspectiva de la relación entre dos focos: el gobierno de los otros, esto es, las artes de conducir la conducta de aquellos a quienes se gobierna; y el gobierno de sí, es decir, el arte de gobernar autónomamente la propia conducta. Es notable que sea justo en este periodo que Foucault retome el concepto de crítica y su relación con la Aufklarüng kantiana, pues, como lo dice en el curso de 1982-1983, el estudio de estos conceptos plantea precisamente el problema de la relación entre el gobierno de sí y el gobierno de los otros (Foucault, 2011a, p. 16). Cabe recordar, con Castro-Gómez (2016a, pp. 12-15), que a partir de 1978 Foucault dio un giro hacia el modelo gubernamental de análisis del poder abandonando con ello el modelo bélico, pues aquel le permitía entender las relaciones de poder como conjuntos estratégicos de acciones sobre acciones, que consideran la libertad de actuar de los individuos, y, por tanto, como conjuntos dinámicos, reversibles, modificables, nunca clausurados del todo. Con ello, el autor francés sorteaba serios interrogantes políticos sobre la posibilidad y la efectividad de las prácticas de resistencia, sublevación o contraconducta, mediante la introducción de una 
dimensión irreductible a los ejes del saber y el poder, que habían ocupado el primer plano en sus trabajos anteriores. Me refiero al eje de la subjetivación, preocupación central para Foucault en sus conferencias, cursos y libros de los años ochenta. La introducción de este eje le permitió concebir las relaciones de poder como juegos estratégicos en los que el sujeto puede intentar revertir, confrontar o escapar a los dispositivos que pretenden gobernar su conducta.

En este contexto, la noción de crítica le sirve a Foucault para inscribir su propio trabajo en una ontología crítica de nosotros mismos, concebida "como una actitud, un ethos, una vida filosófica, en la que la crítica de lo que somos es a la vez análisis histórico de los límites que nos son impuestos y prueba de su posible transgresión" (Foucault, 2013b, p. 97). En este sentido, para él la crítica sería una interrogación sobre quiénes somos en el presente y, a la vez, un trabajo sobre nosotros mismos en el ejercicio de nuestra libertad, y marca una apertura a la cuestión de cómo no ser gobernados, en el marco de los procesos de gubernamentalización creciente que experimentamos.

En su conferencia de 1978, titulada ¿Qué es la crítica?, Foucault sitúa la actitud crítica como compañera y adversaria a la vez de las artes de gobernar. Es decir, no la ubica por fuera del movimiento de gubernamentalización, surgido a partir del siglo XVI, sino que la entiende como una de sus líneas de desarrollo. La actitud crítica, en la que Foucault inscribe su trabajo, aparece como un modo de desconfiar, recusar, limitar, transformar, desplazar o intentar escapar a las artes de gobierno de los otros. Al tiempo que se intensifica la cuestión de cómo gobernar a otros, surge la cuestión de "cómo no ser gobernado de esa forma, por ese, en nombre de esos principios, en vista de tales objetivos y por medio de tales procedimientos, no de esa forma, no para eso, no por ellos" (Foucault, 2013a, pp.7-8). Si la gubernamentalización es el movimiento por el cual se configura una relación de sujeción de la conducta a través de un conjunto de técnicas de saber-poder, "la crítica será el arte de la inservidumbre voluntaria, de la indocilidad reflexiva", el arte de la desujeción a través de un conjunto de técnicas de gobierno autónomo de la propia conducta (Foucault, 2013a, pp. 10-11).

Es notable que Foucault utilice el concepto de arte, pues esto indica que la crítica no es una fría evaluación de argumentos, sino un conjunto de prácticas, de técnicas, que se ejercitan en la producción de un ethos, de un modo de ser o una manera de vivir. La evaluación de argumentos podría ser apenas una práctica parcial perteneciente a un arte del hacer y decir de otro modo. Como sugerí antes, la crítica involucra el gobierno de la propia conducta en una relación dinámica y conflictiva con las técnicas dominantes de producción de la vida; de ahí que no se presente como un afuera absoluto de las relaciones de saber-poder, sino como una actitud límite. En este sentido, el discurso crítico forma parte de ensayos de desujeción o ejercicios de experimentación y desplazamiento de las formas de vida impuestas a los sujetos, que exceden el plano discursivo e implican otros aspectos del ethos, como el uso del tiempo, las formas de alimentación, las relaciones sexuales, de amor y de amistad, entre otros. Es lo que Foucault llamaba estética de la existencia, hacer de la propia vida la producción continua de una obra de arte².

En este punto es relevante aproximarse a la relación entre el concepto de crítica y la Aufklarüng kantiana. Un primer aspecto que me parece importante destacar es la forma de aproximación de Foucault a la pragmática discursiva del texto de Kant sobre la Ilustración. Esta forma de aproximación toma el texto de Kant como una respuesta performativa a la cuestión planteada; es decir, un discurso que no solo enuncia cómo entender el concepto, sino que constituye un auténtico ejercicio de Aufklarüng, principalmente por dos razones: a) por la aparición del texto en una revista dirigida al público, esto es, por estar en juego el uso público de la razón en un medio de circulación abierto al profano; b) porque la cuestión que plantea en un medio de circulación periódico es justamente el sentido y la pertenencia a un presente: “ $¿ Q u e ́$ pasa hoy?, ¿qué pasa ahora?, ¿qué es ese 'ahora' dentro del cual estamos unos y otros, y que es el lugar, el punto [desde el cual] escribo?" (Foucault, 2011a, p. 21). En el texto de Kant, por primera vez según Foucault, la filosofía se interroga por la pertenencia a un nosotros, de manera que el individuo mismo que escribe a un público se sitúa con respecto a ese presente del que forma parte y se pregunta cómo tiene que "desempeñar cierto papel en ese proceso, en el que resultará a la vez, por tanto, elemento y actor" (Foucault, 2011a, p. 22). En síntesis, la pragmática del discurso de Kant nos señala el alcance y los límites de la Aufklarüng, en la forma de una divisa, un acto de habla que, como lo sugiere Foucault, es a la vez un signo de pertenencia y una consigna, un trabajo que hay que hacer: "Ten el coraje de pensar por tu cuenta y de decir todo cuanto pienses, siempre y cuando se trate de un uso público de la razón". Para el Kant de Foucault, los márgenes críticos de la Aufklarüng se marcan en esta distinción entre el uso público de la razón, en donde se exige el coraje de pensar por cuenta propia y decir verazmente lo que se piensa, y el uso privado de la razón, en donde se exige "funcionar como una

2 Para revisar en detalle este concepto, véase Santiago CastroGómez (2016b). 
pieza de una máquina". Se sigue que si la crítica es una interrogación sobre los límites de lo que somos (pensamos, decimos y hacemos), en su texto Kant se plantea una pregunta crítica: si pertenecemos a un proceso de Ilustración, ¿hasta dónde y de qué manera es lícito llevar a cabo este proceso? Su distinción entre los dos usos de la razón sería una forma de responder a esta pregunta crítica.

Por otro lado, considero importante destacar, para efectos de este artículo, la atención que presta Foucault a la noción de salida (Ausgang) presente en la definición de la Aufklarüng como salida de la minoría de edad. Salida señala un desplazamiento hacia otro lado, un umbral que es preciso cruzar. Definir la Ilustración en términos de salida es una forma de responder quiénes somos en términos de algo que estamos dejando de ser y de algo que adviene o a lo que aspiramos. Es notable, de nuevo, la relación con el concepto de crítica antes esbozado: la crítica es una actitud límite, un trabajo en las fronteras de lo que somos, en el umbral que marca nuestra pertenencia a un presente. Se ejerce la crítica para señalar aquello que estamos dejando de ser, en vista de un porvenir, vislumbrar caminos y obstáculos para ser de otro modo, probar franquear los límites que se nos han impuesto. La salida de la minoría de edad es, así, un proceso continuo de reflexión e indocilidad frente a lo que se nos impone como presente, antes que un estado logrado sobre el que no hay vuelta atrás. Para retomar lo dicho al inicio de este apartado, Foucault (2011a, p. 38 ) interpreta la Aufklarüng kantiana como un proceso en el que se supera un déficit de gobierno de sí en relación con el gobierno de los otros, donde los sujetos se desprenden del uso meramente privado de la razón y se atribuyen el derecho a cuestionar las doctrinas, los dogmas y el funcionamiento de las instituciones que nos gobiernan. La minoría de edad, desde esta perspectiva, es el resultado de un exceso de gobierno de los otros tras los límites de lo que corresponde al gobierno de cada uno. Y la crítica aquí desempeña un papel central, por cuanto se encarga de reconocer estos límites y, por tanto, estos excesos de las artes de gobierno de la conducta, y se arroja, en un acto de coraje, a restablecer los espacios para la autonomía frente a los modos de hacer y de decir arrebatados a los sujetos por la gubernamentalidad moderna.

Aclarar este concepto de crítica ayuda a esclarecer el aporte de una perspectiva foucaultiana a un análisis discursivo. Es cierto que Foucault no puede considerarse sin más un analista del discurso, pero también es cierto que sus investigaciones genealógicas requirieron análisis discursivos en algunos momentos. Por ejemplo, en Seguridad, territorio, población, Foucault (2011b) sugiere lo que podría tomarse como un análisis discursivo desde una perspectiva genealógica. Allí afirma que un análisis de este tipo se realiza con el fin de "reconstruir el funcionamiento del texto no desde el punto de vista de las reglas de formación de los conceptos, sino de los objetivos, las estrategias a las cuales obedece y los programas de acción política que sugiere" (p. 56). Para el analista, el texto, considerado como práctica discursiva, remite a otras prácticas (discursivas y no discursivas) que se articulan con él y permite reconstruir la red de elementos que se vinculan en vista de un mismo objetivo estratégico (dispositivo). En esta reconstrucción intervienen tres aspectos que resultan cruciales para un diagnóstico crítico de nuestro presente: la aparición de ciertos saberes considerados científicos, las técnicas y mecanismos de poder, y, en correlación con los otros dos aspectos, la aparición histórica de cierto tipo de sujeto.

Por su parte, Fairclough sostiene que el análisis crítico del discurso investiga cómo las prácticas discursivas y los textos determinan y son determinados por relaciones de poder y luchas por el poder (Fairclough, 1993, p. 135) ${ }^{3}$. De manera similar, en una perspectiva foucaultiana se pueden analizar los discursos como prácticas inmanentes a relaciones de poder. De ahí que se enfaticen los condicionamientos de las prácticas discursivas en las redes de poder de las que forman parte. Pero la existencia de tales condicionamientos no significa que los sujetos hablantes sean completamente ciegos y pasivos frente a relaciones de fuerza que los determinan por completo y los sobrepasan. Sus prácticas discursivas son también, muchas veces de manera deliberada, determinantes para resistir, confrontar o reconfigurar las relaciones de poder en las que están inmersos. Justamente esa sería la función de la crítica: hacer el diagnóstico de las relaciones de poder, mostrar sus límites y los modos de franqueamiento posible.

Fairclough distingue entre texto y práctica discursiva, pues el texto es la instancia de análisis configurada en una práctica discursiva y la práctica discursiva, "la producción, distribución y consumo de un texto" (Fairclough, 1993, p. 138). Un análisis crítico del discurso, según él, se centra en los eventos discursivos, es decir, instancias de uso del lenguaje, analizadas como texto, práctica discursiva y práctica social (Fairclough, 1993, 138). De ahí que el análisis del texto se haga en función del análisis de sus condiciones de producción, circulación y consumo, que están involucradas en procesos o problemáticas sociales. Desde una perspectiva foucaultiana, se puede

3 Más adelante, precisaré la distinción entre práctica discursiva y texto, que tomo de Fairclough. 
afirmar que cuando se interpreta un discurso como una práctica se está analizando cómo se hace el texto (condiciones de producción) y lo que este hace (efectos producidos en su circulación y uso). Dado que se dirige a dilucidar los condicionamientos del texto en los juegos de poder en los que está inmerso, este tipo de análisis pretende descifrar el orden del discurso en el que una práctica discursiva se ha hecho posible. Fairclough entiende el orden del discurso como la "totalidad de prácticas discursivas de una institución y las relaciones entre ellas" (Fairclough, 1993, 138). Desde el punto de vista de Foucault, se podría agregar que esas relaciones entre las prácticas discursivas son inmanentes a relaciones de poder. Entonces, a partir de una interpretación de la lección inaugural de Foucault en el Collège de France (Foucault, 1992), entiendo aquí orden del discurso como el conjunto de prácticas y relaciones de poder ejercidas sobre los discursos, y a través de ellos, que condicionan su producción, circulación y consumo en un ámbito institucional específico.

Lo anterior implica un análisis del modo en que se ejercen relaciones de poder para conjurar los poderes y peligros del discurso, "dominar el acontecimiento aleatorio y esquivar su pesada y temible materialidad" (Foucault, 1992, p. 11). Desde una perspectiva foucaultiana, el orden del discurso se dirige a controlar poderes y peligros asociados a las prácticas discursivas en su materialidad: su heterogeneidad, el hecho de que proliferen sin cesar y por todas partes, de que puedan circular y ser usadas por muchos y de muchos modos, de que en ellas resida una potencia de transformación política. El orden del discurso es garantizado por unos procedimientos (discursivos y no discursivos) para excluir (e incluir), identificar y distribuir los discursos, de manera que algunos sean visibles y otros no, unos sean normales y otros anormales, se puedan garantizar unas identidades y unas jerarquías entre ellos, y que, de este modo, cada uno esté en su lugar, circulando y usándose de una manera determinada.

Esta opción metodológica contribuye, asimismo, a evitar la suposición de un poder entendido como dominación clausurada, "a título de dato fundamental, de principio único, de explicación o ley ineludible" (Foucault, 2013a, p. 33), pues el análisis se encamina a restituir las condiciones de un acontecimiento histórico a partir de un conjunto de elementos heterogéneos y de interacciones que guardan unos márgenes de incertidumbre (Foucault, 2013a, pp. 32-33). A esto apunta Foucault cuando se refiere a la ontología crítica de nosotros mismos como una investigación genealógica en su finalidad y arqueológica en su método. Arqueológica en el sentido de que se ocupa de "los discursos que articulan lo que pensamos, decimos y hacemos, como otros tantos acontecimientos históricos"; y genealógica en el sentido de que

[...] no deducirá de la forma de lo que somos lo que nos es imposible hacer o conocer; sino que extraerá, de la contingencia que nos ha hecho ser lo que somos, la posibilidad de no ser, no hacer, o de no pensar, por más tiempo, lo que somos, lo que hacemos o lo que pensamos. (Foucault, 2013b, p. 91).

Pues bien, este concepto de crítica y estas opciones metodológicas, ¿cómo encaminaron el análisis crítico del discurso realizado? Lo primero sería reconocer que la crítica ejercida no se ha hecho a distancia, por fuera de las relaciones de gubernamentalidad articuladas a los discursos predominantes en la actualidad. Por el contrario, es preciso aceptar que este ejercicio crítico se ha pensado y se ha escrito ya dentro de unas condiciones discursivas atravesadas por aquellas relaciones de poder. Por ejemplo, por unas redes institucionalizadas de control de la escritura académica o por unos procedimientos de autorización y disciplinarización del saber. Lo segundo sería reconocer que la crítica se torna autocrítica, en la medida en que quien escribe estas líneas, por cuanto forma parte del sistema de enseñanza universitaria, participa de algún modo en el funcionamiento de este ensamblaje de discursos que pretenden direccionar las prácticas de formación y evaluación en las universidades. Y siendo de este modo autocrítica, ha buscado mostrar la contingencia de esas prácticas de gobierno que se han naturalizado en las universidades, encontrar sus excesos y las herramientas para desgubernametalizar las prácticas educativas. En este sentido, los textos analizados fueron tomados como instancias que buscan direccionar las prácticas pedagógicas de los maestros y, en consecuencia, comunicar cómo han de ser conducidas las relaciones de gobierno de la conducta de los estudiantes; de ahí que sirvan para entender quiénes somos los maestros universitarios en la actualidad colombiana. Pero también fueron asumidos como fuentes que proporcionan herramientas para re-conocernos como sujetos de otros modos de hacer y de decir, esto es, para posibilitar modos de reconocimiento de las prácticas de indocilidad reflexiva pertinentes a las condiciones actuales de las instituciones universitarias.

\section{Un discurso moderno y crítico}

El análisis discursivo realizado se centró en un momento que representa la apertura a una preocupación singular: las competencias genéricas de los estudiantes universitarios en Colombia. Este momento puede 
ubicarse, por un lado, entre 2003 y 2009 , cuando el Estado está preocupado por normalizar prácticas de evaluación de competencias genéricas mediante exámenes estandarizados; por otro lado, de 2009 a la actualidad, cuando las universidades responden a estas medidas con estrategias pedagógicas para mejorar sus resultados en las evaluaciones y la calidad del servicio que prestan. En este marco, surgen una serie de documentos gubernamentales que buscan brindar lineamientos para que las prácticas educativas en las universidades se ajusten al discurso de las competencias. En la investigación que dio origen a este artículo de reflexión se tomaron algunos de estos documentos para un análisis textual más riguroso ${ }^{4}$, entendiendo que la introducción de la evaluación de competencias genéricas por parte del Estado consolida definitivamente el discurso de las competencias en la educación superior.

La primera conclusión que quisiera abordar, a partir del análisis discursivo realizado, es que este discurso de las competencias genéricas se presenta como una reflexión pertinente y necesaria frente a las condiciones de la sociedad actual y, además, se muestra favorable a la formación de sujetos críticos y autónomos. ¿Se sigue que este discurso es una manifestación del movimiento de Ilustración que exponíamos en el apartado anterior? Si acogemos la definición de modernidad planteada por Foucault, como una actitud frente al presente que representa una pertenencia y una tarea por hacer (Foucault, 2011a, p. 81), ¿ el discurso de las competencias es un discurso moderno en este sentido? $i$ El gobierno del sistema de educación superior por fin se ha encaminado en la vía de la emancipación de los ciudadanos?

Primero abordaré esta insólita similitud entre la modernidad foucaultiana y el discurso pedagógico de las competencias genéricas en la educación superior colombiana. Para ello, prestemos atención a la pragmática del discurso analizado, tal como Foucault hace con el texto de Kant. Esa pragmática indica dos cosas: la primera es que el discurso se presenta como elemento y actor de su propia actualidad. Su justifica-

4 Cabe aclarar que en el análisis crítico del discurso, a diferencia de las aproximaciones genealógicas y arqueológicas, no se habla de construcción de archivo, sino de selección de corpus. Por su enfoque metodológico, un análisis de este tipo no se basa necesariamente en una argumentación inductiva a partir de una muestra representativa, sino de una selección estratégica de textos que permitan establecer relaciones con el contexto discursivo analizado. En nuestro caso, los documentos seleccionados para el análisis textual fueron: la Propuesta de lineamientos para la formación por competencias en educación superior (MEN, 2011); el Decreto 3963 de 2009. Por el cual se reglamenta el Examen de Estado de Calidad de la Educación Superior (MEN, 2009a), y el Boletín Informativo No. 13. Educación superior (MEN, 2009b). ción apela a una reflexión sobre el presente: ¿En qué consiste este presente social en el que vivimos? Para responder a esta pregunta, el Estado periódicamente convoca a unos expertos en educación y pedagogía, que le asesoran en la producción de estándares o lineamientos en competencias, basados en un estado del arte y una reflexión sobre ese presente social.

Una identificación inicial de competencias genéricas para educación superior en el país, se elaboró a partir de una reflexión por parte de reconocidos miembros de la comunidad académica nacional, convocados por el MEN, quienes revisaron el estado del arte en esta materia y seleccionaron aquellas competencias que responden a las exigencias que demanda la sociedad de la formación profesional. (MEN, 2011, p. 1).

Esta reflexión sobre nuestro presente señala, en efecto, una pertenencia, pero también una consigna, al modo de lineamientos dirigidos a las universidades y los profesores. Estos lineamientos no solo describen unas competencias, sino que se tornan prescriptivos de las prácticas educativas; representan una definición de lo que es pertinente y constituye la calidad de la formación brindada por las universidades.

Cada vez se reconoce más la necesidad de una formación en educación superior que sea pertinente para la sociedad, por ello la formulación de competencias genéricas, que derivan en otras más especializadas, constituye el horizonte de acciones de formación deseables en educación superior y a la vez son un referente de gran importancia para poder monitorear la calidad de la formación en todos los programas académicos de pregrado. (MEN, 2011, p. 1).

En dichos lineamientos está en juego el gobierno de los otros, en este caso las prácticas educativas de maestros y estudiantes.

El otro aspecto de la pragmática discursiva de los textos es que profieren las prescripciones a esos otros desde un umbral crítico, un movimiento de salida (Ausgang). Esto implica que la pertenencia del discurso al presente está definida por el intervalo entre aquello que hay que dejar atrás y aquello a que aspiramos. Esto se evidencia en una estrategia discursiva común en los discursos de renovación pedagógica, como el discurso de las competencias, a saber, la de identificar la vieja escuela o la educación tradicional con lo caduco, lo que no es pertinente o no tiene la calidad esperada.

Partiendo de dicho diagnóstico, una Comisión se propuso identificar las competencias necesarias en el campo laboral que se enseñaban precariamente en la escuela; justificar por qué dichas habilidades 
eran indispensables y comunicar los resultados a las comunidades académicas y corporativas. (MEN, 2009b, p. 4).

Frente a estos viejos modelos, el discurso de las competencias busca decir y hacer de otro modo en la educación superior. Lo dicho se puede articular a la idea, antes sugerida, de que el Estado convoca al experto para que su reflexión refleje el presente y esto justifique sus direccionamientos. Ya no es, pues, una tradición, unas costumbres o un conjunto de doctrinas lo que pretende legitimar la calidad de una formación, sino la actualidad económica y social, que exige al Estado monitorear el tipo de formación ofrecida a la población. Y si este discurso es un reflejo del presente y allí está en juego el gobierno de las prácticas educativas, este se convierte en un espejo para las instituciones y los maestros, en una consigna ilustrada para que conduzcan sus prácticas educativas según este reflejo.

¿Cómo es que la divisa ilustrada y la actitud crítica terminan funcionando en los discursos que pretenden gobernar nuestras prácticas educativas? Si la crítica surge como una línea de desarrollo de la gubernamentalidad, según mostré en el apartado anterior, no es del todo sorprendente que forme parte de esos discursos que pretenden gobernarnos. El discurso de las competencias se justifica por un llamado a que el sistema educativo se haga pertinente frente al reflejo del presente social y económico que diagnostican los expertos, hombres ilustrados que conocen ese presente y asesoran al Estado en calidad de tales. Se puede entender como un llamado a las instituciones y los maestros a salir de los viejos modelos que van en contra de la formación de sujetos críticos y autónomos. Pero este llamado no debe prestarse a confusiones: es un llamado a aceptar el presente y someterse a él, mientras que la crítica era definida por Foucault como el arte de la inservidumbre voluntaria. Es preciso, entonces, asumir una posición crítica frente a esta gubernamentalización de la crítica, esto es, mostrar también sus excesos y sus límites en tanto ejercicio del poder, para poder vislumbrar unos umbrales de salida.

Para empezar, es útil preguntar cuál es exactamente el presente que se refleja en el discurso de las competencias en la educación superior. Sostengo que este presente no representa una salida emancipatoria de nuestras actuales servidumbres, sino el umbral entre las sociedades disciplinarias, en crisis, y las sociedades de control, en maduración. En este punto es útil retomar el postscriptum sobre las sociedades de control de Deleuze (2006), que nos sitúa en el devenir entre estos dos tipos de regímenes sociales.
Los regímenes disciplinarios operan mediante la organización de grandes centros de encierro, en los que

[...] el individuo pasa de un círculo cerrado a otro, cada uno con sus propias leyes: primero la familia, después la escuela ("ya no estás en la casa"), después el cuartel ("ya no estás en la escuela"), a continuación la fábrica, cada cierto tiempo el hospital y a veces la cárcel, centro de encierro por excelencia. (Deleuze, 2006, p. 2).

Los regímenes de control, en cambio, se identifican con "formas ultrarrápidas que adopta el control 'al aire libre' y que reemplazan a las antiguas disciplinas que actuaban en el periodo de los sistemas cerrados" (Deleuze, 2006, p. 2). En estas formas de control abierto no hay un comienzo desde cero en el paso de un centro de encierro a otro, sino una línea continua que los atraviesa. "Los encierros son moldes o moldeados diferentes, mientras que los controles constituyen una modulación, como una suerte de molde autodeformante que cambia constantemente y a cada instante" (Deleuze, 2006, pp. 2-3).

De ahí que la vieja analogía escuela-fábrica-cárcel dé paso a la formación permanente, la mejor forma de poner "la escuela en manos de la empresa" (Deleuze, 2006, p. 3). No es, pues, que la universidad sea análoga a una empresa, sino que la universidad forma un continuum con la empresa. Las empresas se empiezan a ocupar cada vez más de la formación de sus empleados y las universidades se preocupan por la adecuada inserción de la población en un mercado laboral mundial y cambiante. En estas condiciones, la universidad entra en la lógica empresarial, que utiliza más los estímulos para la modulación de los salarios que los castigos correctivos por las faltas cometidas y necesita empleados más flexibles y autónomos; y esta lógica invade las relaciones pedagógicas.

La extensión de la lógica empresarial a las relaciones pedagógicas tiene que ver con un cambio en el tipo de economía preponderante, que en los textos analizados aparece enunciada como una economía basada en el conocimiento y una internacionalización de las economías.

El Consejo ha propuesto a los países miembros un marco de Competencias clave que contribuirán a lograr "una economía basada en el conocimiento, con más y mejores empleos y cohesión social". Este mandato político se constituye en la respuesta del Consejo a las aceleradas transformaciones del mundo laboral, como resultado de la internacionalización de las economías, la globalización y la falta de mano de obra calificada para responder a las nuevas demandas. (MEN, 2009b, p. 5). 
Ese tipo de economía modifica las formas del saber y, con ello, las de organización del currículo, los roles asignados a docentes y estudiantes, y las prácticas de enseñanza y de aprendizaje.

La frontera entre los saberes es cada vez más tenue, por ejemplo, cuando se habla de biotecnología se mezclan Medicina e Ingeniería. Es decir, el conocimiento no es un contenido que se puede empaquetar en un contenedor, es un tejido de saberes que permite generar un nuevo conocimiento, el cual, siendo así, resulta infinito. Es por ello que las universidades se están viendo abocadas a transformar sus currículos. (MEN, 2009b, p. 3).

El saber se presenta como una red infinita y abierta, una línea autodeformante que se transforma a un ritmo acelerado y que atraviesa las fronteras disciplinares y territoriales del conocimiento. En consecuencia, los currículos actuales no han de compartimentar el conocimiento en disciplinas, sino que se deben tornar interdisciplinarios, y su objetivo no debe ser tanto impartir conocimientos estancos a los estudiantes, sino facilitar la construcción del conocimiento que a estos les resulte más útil con respecto a un mercado laboral cambiante.

En el mundo se han identificado algunas competencias clave para el aprendizaje permanente que deben llevar a las instituciones educativas a propiciar en sus ambientes académicos los contextos necesarios para el aprendizaje durante toda la vida, además de las conexiones necesarias entre lo que aprenden en la universidad y su aplicación en los distintos campos de acción profesional. (MEN, 2011, p. 18).

El tipo de prácticas formativas para este tipo saber debe estimular un ejercicio de aprendizaje lo más real posible. Tendemos, entonces, a los simuladores, que permitan aprender de experiencias significativas. Si Deleuze veía en los concursos televisivos una expresión de la modulación de los salarios a través de estímulos o incentivos, me atrevería a decir que los realities de hoy son expresión de la difuminación de las fronteras entre la ficción y la realidad, que es propicia para generar aprendizajes significativos, aprendizajes para la vida (laboral). Como decía antes, la escuela no es análoga a la fábrica, sino que las instituciones de educación superior trazan una línea continua con las empresas, simulándolas, de manera que estén en capacidad de facilitar los tránsitos de una empresa a otra, las prácticas reales y, con ello, una formación pertinente y para la vida.

El maestro, más que un erudito, ha de ser un sujeto competente, es decir, debe poseer un conocimiento aplicable y flexible: capacidad de atravesar las disciplinas, apropiarse aquí y allá del conocimiento, interactuar en contextos cambiantes y adaptarse a nuevas tareas que van saliendo en el proceso, pues se ha convertido en un facilitador y supervisor de los procesos de aprendizaje de los estudiantes. Sus acciones tienden cada vez más a estar definidas como gestión (de currículo, de aprendizaje, de investigación), lo que le lleva a ocupar su tiempo en producir informes, cuadros, tablas, indicadores y balances. Por su parte, los sujetos en formación que requiere la nueva economía han de ser, asimismo, sujetos competentes, dadas las condiciones cambiantes e inciertas que definen el presente social. De ahí que el discurso de las competencias se muestre favorable a la formación de sujetos críticos: capaces de cuestionar los saberes existentes y producir nuevos conocimientos, es decir, reflexionar sobre los límites de su presente e ir más allá de ellos.

El aprendizaje para toda la vida, la comprensión de contextos y situaciones que exige la toma de decisiones argumentada, las posibilidades de análisis y de crítica ante diversos enunciados, se han identificado como competencias que deben ser fuertemente desarrolladas ante el cambiante estado de las tecnologías de la información y la comunicación y el vertiginoso avance de los conocimientos sobre aquellos aspectos que demanda la sociedad de los futuros profesionales, universitarios, técnicos o tecnólogos. (MEN, 2011, p. 1).

También, deberán ser sujetos autónomos: capaces de resolver problemas por sí mismos, tomar decisiones argumentadas, aprender por su propia cuenta, mantenerse actualizados y comunicarse asertivamente con el público al que se dirigen sus productos.

El desarrollo de herramientas de metacognición y de versatilidad del conocimiento que soportan la capacidad de seguir avanzando en el aprendizaje de forma autónoma y la capacidad de transferir conocimientos y competencias adquiridos en un determinado contexto a contextos distintos, son factores esenciales para el despliegue de las competencias arriba mencionadas. (MEN, 2011, p. 18).

Considero que estas subjetividades trazadas por los lineamientos de competencias pueden vincularse al pensamiento y el programa del neoliberalismo norteamericano, analizado por Foucault en las clases finales de Nacimiento de la biopolítica. Los neoliberales dirigen su atención a la educación superior, por ser este el lugar idóneo para la acumulación de capital humano. En Nacimiento de la biopolítica, Foucault (2007) muestra que el pensamiento neoliberal, al desplazar la noción de capital hacia la conducta económica individual y entenderla en términos de todo aquello que le genera al trabajador una renta 
futura, define el concepto de capital humano como la acumulación de capacidades (competencias) que hace un individuo a lo largo de su vida con el fin de generar rentas futuras. Según la lectura de Foucault (2007), un capital es

\section{[...] el conjunto de los factores físicos, psicológicos, que otorgan a alguien la capacidad de ganar tal o cual salario, de modo que, visto desde el lado del trabajador [...], el trabajo comporta un capital, es decir, una aptitud, una idoneidad; como suelen decir, es una "máquina”. (p. 262).}

Este desplazamiento de la noción de capital trae dos consecuencias que me parecen interesantes para efectos del análisis: la primera es el intento de "desproletarizar" de la sociedad. Al hacer del trabajador un homo oeconomicus que, en su conducta individual como trabajador, calcula costos y beneficios, y acumula capital humano 5 , "es el propio trabajador quien aparece como si fuera una especie de empresa para sí mismo" (Foucault, 2007, p. 264). Esto trae consigo la concepción de la economía como si estuviera constituida por unidades-empresa, que compiten y cooperan entre sí, antes que capitalistas que, al explotar a los trabajadores, acumulan capital. La segunda consecuencia es la extensión de la forma-empresa a todos los ámbitos de la vida social. Los neoliberales utilizaron la figura del homo oeconomicus como grilla de análisis para todas las relaciones sociales (matrimonio, maternidad, educación, etc.):

\begin{abstract}
Generalizar efectivamente la forma 'empresa' dentro del cuerpo o tejido social; quiere decir retomar ese tejido social y procurar que pueda repartirse, dividirse, multiplicarse no según la textura de los individuos, sino según la textura de la empresa [...]. Y es necesario que la vida misma del individuo [...] lo convierta en una suerte de empresa permanente y múltiple". (Foucault, 2007b, p. 277).
\end{abstract}

Esta idea neoliberal se puede evidenciar en lo que sugerí antes en torno a los currículos y las prácticas pedagógicas: la forma-empresa invade las relaciones pedagógicas de las universidades.

Si es esta grilla de análisis neoliberal la que está en el trasfondo de la formación permanente ${ }^{6}$, podría decirse que las universidades adquieren la forma de una empresa y, a su vez, los individuos serían

5 "Es importante reconocer que estas cuatro competencias genéricas son definitivas, y se acercan al ideal de capital humano que requieren los sectores productivos". (MEN, 2009b, p. 13).

6 "Vale la pena resaltar que las competencias genéricas que se desarrollan en la educación superior acompañarán al estudiante toda la vida en el desempeño que este tenga en la sociedad". (MEN, 2009b, p. 13). unidades empresariales que invierten parte de sus ganancias en, o bien se endeudan para, capitalizarse a través del servicio que ofrecen las universidades. En esa relación económica, el Estado, que gobierna según una agenda económica mundial, cumple el papel de organismo que controla la calidad del servicio educativo, con el fin de garantizarle a la población, y al mercado laboral mundial, una acumulación de capital humano que sea pertinente para las dinámicas de ese mercado. Como esas dinámicas están sujetas a cambios acelerados, en relación con el desarrollo abierto e igualmente acelerado de tecnología y conocimiento, y se dan en un marco de intercomunicación y quiebre de las fronteras, el control de calidad ejercido por el Estado ya no se dirige a comprobar únicamente conocimientos específicos de las distintas profesiones, sino a establecer unos estándares de competencias genéricas que deben desarrollar las organizaciones universitarias. Por supuesto, en este mundo intercomunicado y abierto esos estándares están alineados con los estándares de competencias genéricas formulados en los centros económicos y políticos mundiales ${ }^{7}$.

¿Cómo entender, entonces, que el Estado colombiano y las universidades enfoquen sus políticas en la formación de sujetos autónomos y críticos? Como lo afirma Castro-Gómez (2016a),

[El neoliberalismo] abre la posibilidad de un sujeto activo, responsable, competitivo, capaz de gobernarse a sí mismo sin tener que recurrir a la protección ofrecida por los aparatos estatales de gobierno. La autodeterminación del sujeto se convierte de este modo en la clave para la prosperidad económica de la sociedad. (p. 25).

Pero la consigna ilustrada de la que hablábamos antes no se dirige así a la emancipación respecto de la gubernametalización de la subjetividad, sino a nuevos modos de gobierno heterónomo de la subjetividad, a través de formas de control abierto.

7 En este punto quiero remitir al lector al artículo de Doris Santos (2013). Allí ella afirma que el discurso de la formación por competencias constituye un nuevo lenguaje que los miembros de las universidades latinoamericanas se han visto abocados a adoptar. Este lenguaje es ideológico en la medida en que se presenta como la única realidad posible ante las circunstancias de la educación superior en la región y conlleva una especie de mímesis, en la que las universidades latinoamericanas deben seguir pasivamente los pasos y aprendizajes de los países "más desarrollados". Un ejemplo que apoya esta afirmación es el uso de la metáfora de la sintonía (tune, en inglés; de ahí viene el nombre del proyecto Tuning), que es una manera en la que el discurso analizado intenta hacer una concesión a la diversidad, pero en términos de la afinación de los instrumentos para interpretar la música sin disonancias: "La imitación de una melodía compuesta en otras latitudes (adopción de un sistema de ideas foráneo)" (Santos, 2013, p. 214). 


\section{Poder disciplinario en la educación superior colombiana}

Hasta el momento habíamos fijado la atención en el modo en que el discurso de las competencias en la educación superior colombiana define y prescribe al sujeto competente como un sujeto crítico y autónomo, por cuanto estas cualidades son funcionales a los regímenes de control y la economía política neoliberal. Se supone que este discurso refleja también una disposición moderna y crítica, en la medida en que se basa en una pretendida reflexión sobre el presente y busca una salida de los viejos regímenes disciplinarios. No obstante, cuando se fija la mirada en los efectos y los modos específicos de operar del discurso, es notable que este forma parte de un conjunto de procedimientos de corte más bien disciplinario. El lenguaje de las competencias adquiere eficacia precisamente a través de la legislación sobre los exámenes de Estado y las políticas de calidad educativa. Y estos vienen a reforzar el poder disciplinario en la educación superior, antes que a convertirlo en un hecho del pasado. ¿Cómo es que un discurso del dejar actuar la libertad y la autodeterminación de los sujetos se efectúa a través de técnicas disciplinarias como el examen? ¿Cuáles son las implicaciones de esta articulación entre poder disciplinario y gubernamentalidad neoliberal? Para abordar estas preguntas, en este apartado exploraré el otro lado del umbral que define el presente de nuestras prácticas educativas en la educación superior: el del poder disciplinario.

En Foucault, el poder disciplinario se refiere a un conjunto de técnicas sobre el cuerpo humano que lo hacen, en el mismo mecanismo, tanto más obediente cuanto más útil, y viceversa (2009, p. 160). Esto es lo que él llama una anatomía política dirigida a la fabricación de cuerpos dóciles, a través de una disociación de sus fuerzas: "La disciplina aumenta las fuerzas del cuerpo (en términos de utilidad económica) y disminuye esas mismas fuerzas (en términos de obediencia política" (Foucault, 2009, p. 160). La hipótesis que me interesaría explorar es que este esquema de disociación del poder del cuerpo es el que opera en nuestro presente educativo y se puede identificar con la consigna ilustrada señalada por Kant en su texto sobre la Aufklärung: "Razonad cuanto queráis, pero obedeced".

Olvidábamos que la anatomía política del sujeto competente delineada por grupos de expertos se hacía en función del monitoreo de la calidad de la educación, por parte del Estado.

Desde el año 2008 el Ministerio de Educación Nacional ha emprendido acciones tendientes a la formulación de competencias genéricas, o transversales a todos los núcleos de formación en educación superior, que posibiliten un monitoreo de la calidad de la educación superior en el país y que puedan constituirse en el elemento articulador de todos los niveles educativos: inicial, básica, media y superior. (MEN, 2011, p. 1).

Este monitoreo forma parte de una estrategia para articular todos los niveles del sistema educativo colombiano mediante la evaluación de competencias transversales a estos niveles y a los diferentes núcleos formativos disciplinares. Pareciera, nuevamente, una estrategia gubernamental del orden de la formación permanente propia de los regímenes de control. Pero esta estrategia adquiere su eficacia de una técnica eminentemente disciplinaria: el examen, ya que este se constituye como una mirada normalizadora (Foucault, 2009, p. 215). No hay que esperar a los dispositivos de control para presenciar un ejercicio del poder que acompañe los procesos de producción y aprendizaje en toda su duración; la vigilancia jerárquica propia de las disciplinas se encarga de esta tarea de control permanente de la actividad: vigilar pasa a ser "una función [...] que debe formar parte integrante del proceso de producción; debe acompañarlo en toda su duración" (Foucault, 2009, p. 204).

La estrategia de monitoreo del Estado, si bien pretende empresarios de sí mismos, se despliega a través de mecanismos de normalización con fines de inspección y vigilancia. En 2009 el Ministerio de Educación Nacional (MEN) promovió la Ley 1324, por la cual se fijaron parámetros y criterios para organizar el sistema de evaluación de resultados de la calidad de la educación, "en procura de facilitar la inspección y vigilancia del Estado" (Congreso de Colombia, $2009)^{8}$. En esta ley el MEN dio un paso decisivo para reglamentar la evaluación estandarizada, definida en términos de una evaluación "externa e independiente", administrada por el Instituto Colombiano de Fomento de la Educación Superior (Icfes), "comparable

8 En este punto quiero remitir al lector al artículo de Sandra Soler (2011), en el que analiza el Plan decenal de educación 2006-2016, promovido por el gobierno de Álvaro Uribe. Allí la autora señala una posible relación entre el énfasis dado a la evaluación en todos los niveles, que "sea coherente con los estándares de calidad nacionales e internacionales", con uno de los lemas bandera de ese gobierno: la transparencia. "Este concepto, por demás problemático, lleva a que todas las instituciones sean objeto de continua vigilancia mediante las famosas rendiciones de cuentas, que al final no son más que formas solapadas de control, importadas de los sistemas de medición de productividad de las empresas -basadas en la eficacia- que han convertido a los funcionarios de la educación y a los docentes en productores de cuadros, tablas, indicadores, balances e informes que los alejan de las actividades académicas, pero los mantienen ocupados para que no puedan ejercer la crítica y el disentimiento frente a las políticas del Estado" (Soler, 2011, p. 101). 
y periódica". En ese mismo año y en ejercicio de las facultades conferidas en la Ley 1324, el MEN promulgó el Decreto 3963, por el cual se reglamentó el examen de Estado como un "instrumento estandarizado para la evaluación externa de la calidad de la Educación Superior" (MEN, 2009a, Art. 1ํ). El objeto de la evaluación enunciado en este decreto son "las competencias de los estudiantes que están próximos a culminar los distintos programas de pregrado, en la medida en que estas pueden ser valoradas con exámenes externos de carácter masivo, incluyendo aquellas genéricas que son necesarias para el adecuado desempeño profesional o académico" (MEN, 2009, Art. $2^{\circ}$ ). Además de incluir explícitamente la evaluación de competencias genéricas, el decreto reglamentó la responsabilidad de las instituciones de reportar a los estudiantes próximos a culminar sus estudios, so pena de sanciones por parte del MEN.

Lo anterior confirma en el caso colombiano una de las tesis de Foucault en Vigilary castigar (2009, p. 199) y es que las técnicas disciplinarias llegan a invadir poco a poco los aparatos del Estado, "a modificar sus mecanismos y a imponer sus procedimientos" y "el aparato judicial no escapará a esta invasión apenas secreta". Mi hipótesis es que esta invasión de la vigilancia jerárquica y la sanción normalizadora, a través del examen de Estado, refuerza el poder disciplinario en la educación superior colombiana. ¿Cómo ocurre esto?

El poder disciplinario puede entenderse como "el arte del buen encauzamiento de la conducta", "es un poder que, en lugar de sacar y retirar, tiene como función principal 'enderezar conductas', o sin duda, hacerlo para poder retirar mejor y sacar más" (Foucault, 2009, p. 199). Para ello, separa, analiza, diferencia las fuerzas que constituyen a los individuos y pretende multiplicarlas. Los documentos de lineamientos y estándares de competencias producidos por el MEN representan esta mirada analítica sobre el individuo; separan cada una de las competencias que es preciso incrementar y, dentro de esas competencias, diferencian cada uno de los desempeños que permiten medir, calificar y clasificar sus aptitudes. De manera que la crítica y la autonomía, en cuanto competencias categorizadas por desempeños, forman parte de un modelo de sujeto que, como veíamos en el apartado anterior, resulta útil para las relaciones económicas actuales. No se trata de reprimir o prohibir estas fuerzas, sino de encauzarlas hacia su utilidad económica y, al mismo tiempo, desviarlas de sus peligros políticos, por ejemplo, que no se dirijan al progreso de la ciencia y la tecnología o a la productividad de las empresas, sino a la desobediencia frente a las medidas del Estado o las instrucciones de los supervisores.

En esta operación analítica sobre los cuerpos están en juego dos procedimientos que se combinan en el examen: la vigilancia jerárquica y la sanción normalizadora. El examen es "una vigilancia que permite calificar, clasificar y castigar [...], establece sobre los individuos una visibilidad a través de la cual se los diferencia y se los sanciona" (Foucault, 2009, p. 215); de modo que al decretar el examen como instrumento de vigilancia sobre las instituciones educativas, el Estado refuerza esos dos procedimientos.

En primer lugar, analicemos la vigilancia jerárquica. El examen de competencias no solo es una mirada jerárquica de los aparatos del Estado sobre las universidades. Despliega una serie de niveles jerárquicos, una secuencia de heteronomías en cascada. El examen diferencia, mide y compara los resultados de las universidades, establece jerarquías entre ellas en términos de su calidad. Dado su carácter articulador de los niveles educativos, también permite establecer comparaciones entre los desempeños en esos niveles. Cuando un estudiante es diagnosticado con bajo desempeño en una prueba de educación básica, ingresa a la educación superior con un déficit que las universidades buscan corregir. Se construye, entonces, una atribución de culpas y responsabilidades de un nivel a otro: si el estudiante llega con bajas competencias es porque en el nivel anterior no se cumplieron las responsabilidades.

La mirada coactiva del examen de Estado termina diagnosticando también las prácticas de enseñanza de los maestros. Se crea así una mirada de sospecha frente a la autonomía de los maestros, si estos no conducen sus prácticas hacia la mejora de los resultados en las pruebas de Estado. Frente a esta supervisión, el maestro se ve condicionado a asumir el lenguaje y la práctica de fomento de las competencias, según los modelos de desempeño que promueve el MEN, y de aplicar sucesivas pruebas a sus estudiantes, para ir comprobando sus progresos. Por su parte, el estudiante va pasando de un lugar al otro del currículo, de un nivel educativo a otro, con la condición de que obtenga cierto nivel de desempeño en las pruebas. Así, va dejando un registro visible de sus avances, del incremento de competencias o capital humano que ha ido acumulando en su paso por el sistema educativo. No cabe duda de que los exámenes y las calificaciones en las universidades son más viejos que los exámenes de Estado sobre competencias, pero con la introducción de estos pareciera que los maestros y las universidades tienen menos posibilidades de realizar otras prácticas evaluativas. 
En segundo lugar, veamos los efectos del examen en tanto sanción normalizadora. En el poder disciplinario lo que se sanciona es "la inobservancia, todo lo que no se ajusta a regla, todo lo que se aleja de ella, las desviaciones. Es punible el dominio indefinido de lo no conforme [...] la falta del alumno es [...] una ineptitud para cumplir sus tareas" (Foucault, 2009, p. 209). De ahí que se fijen unos estándares, pues estos permiten definir desempeños medibles y, con ello, las desviaciones, las inaptitudes, lo que está por debajo del nivel medio de eficiencia. Pero la sanción no es tanto ejemplarizante o vengativa como correctiva: "El castigo disciplinario tiene por función reducir las desviaciones", por eso los sistemas disciplinarios "privilegian los castigos del orden del ejercicio aprendizaje intensificado, multiplicado, varias veces repetido-" (Foucault, 2009, p. 209). El discurso de las competencias operado bajo exámenes conlleva una retórica del ejercicio, ligada a la del desarrollo: cuanto más se practique, más se desarrolla la competencia. Si la competencia no está desarrollada, entonces no se obtendrá el desempeño que sanciona el examen ${ }^{9}$. Por lo tanto, hay que ejercitarla más. En consecuencia, quienes obtienen bajos resultados son seleccionados y puestos a trabajar más duro en su ejercicio: más tareas, más talleres, tiempo extra, más tutorías. Las universidades crean espacios remediales que terminan convirtiéndose en prolongaciones de la secundaria en el nivel superior. Poco a poco se extienden las estrategias de inmersión en las universidades, los talleres de competencias básicas y las tutorías de acompañamiento para evitar la deserción estudiantil. Se pretende una salida de la minoría de edad de los estudiantes, con más acompañamiento, más diagnósticos y más medidas de encauzamiento de la conducta.

Por otra parte, "el castigo, en la disciplina, no es sino un elemento de un sistema doble: gratificaciónsanción" (Foucault, 2009, p. 210). A través del examen las conductas de los estudiantes, pero también de los maestros y las instituciones educativas, son calificadas a partir de buenas y malas notas. Esto genera una circulación de anticipos y de deudas. No es casual que el momento de los exámenes de competencias sea también el momento de los sistemas de créditos, pues el crédito permite calificar, señala el nivel de credibilidad de un individuo o una institución en términos de sus capacidades (de saber y de prestar un servicio). Esa maquinaria de vigilancia jerárquica, que señalábamos

9 Es cierto que, en tanto modelo pedagógico, el discurso de las competencias generalmente incluye otros instrumentos de valoración y el examen escrito tiene relativa presencia. No obstante, tal como se ha aplicado gubernamentalmente, por lo menos en el caso de la educación superior colombiana, como se ha mostrado hasta acá, se ha dado prelación al examen de selección múltiple. antes, adquiere su concreción en los sistemas de crédito y acreditación en las universidades. Nuevamente, se traza una línea continua entre diferentes ámbitos y niveles: el credo del crédito se extiende desde las agencias calificadoras de las economías nacionales ${ }^{10}$, pasando por las instituciones del Estado que califican la calidad de las universidades y los exámenes de Estado que sancionan a los estudiantes, hasta los pequeños exámenes de cada materia y cada maestro, cada semestre, que registran la acumulación de capital humano. Además, el examen sanciona a través de la distribución según rangos o grados:

Señalar las desviaciones, jerarquizar las cualidades, las competencias y las aptitudes y también castigar y recompensar [...]. La disciplina recompensa a través del juego único de los ascensos, permitiendo ganar rangos y puestos, y castiga haciendo retroceder y degradando. (Foucault, 2009, p. 212).

Los resultados de los exámenes de competencias siempre se sancionan en términos de unos niveles o rangos. Los individuos deben comparar su desempeño individual con unos marcos estándar de desempeño, según los cuales están clasificados en uno u otro nivel. Un mal resultado significa o bien más ejercicio, o bien un estancamiento, la imposibilidad de pasar a otro nivel (de la secundaria a la universidad, por ejemplo); un buen resultado, por el contrario, un ascenso de nivel, una beca, un anticipo, un crédito (pues "ser pilo paga"11).

Con todo, aún podría decirse que estas técnicas disciplinarias contribuyen al incremento y la circulación del pensamiento crítico y la autonomía en nuestra sociedad, y esto podría tener efectos de transformación política. Sin embargo, es importante observar cómo opera el instrumento mismo del examen, para saber bajo qué límites se pretenden desarrollar estas fuerzas. El examen de competencias es un aparato de escritura y el individuo queda sujeto a él como una pieza. En términos generales, el examen sitúa a los individuos "en una red de escritura; los introduce en todo un espesor de documentos que los captan y los inmovilizan. [...] Se conforma

10 En este punto remito al lector al trabajo de Maurizio Lazzarato sobre la economía de la deuda (2013). En este trabajo, Lazzarato muestra cómo la economía de la deuda, propia del neoliberalismo, no acaba con los dispositivos disciplinarios ni con los regímenes de soberanía, sino que los refuerza y los hace funcionales a sus objetivos.

11 "Ser Pilo Paga" es el nombre de un programa del actual Gobierno colombiano, en el que los estudiantes que tengan buenos resultados en las pruebas de Estado a final de la educación media y demuestren bajos recursos económicos, reciben un crédito condonable para estudiar en un programa universitario, con la condición de obtener el título profesional. 
un 'poder de escritura' como pieza esencial en los engranajes de la disciplina” (Foucault, 2009, p. 220). En cuanto a los exámenes de competencias, no solamente generan un extenso archivo de informes que caracterizan el nivel de aptitud de cada cual y la preparación para el mercado laboral de conjuntos enteros de la población -cálculos individuales que repercuten en los cálculos de la población-. Están constituidos ellos mismos como juegos de escritura a la manera de pregunta-respuesta; juegos escritos de interrogación repetida con la finalidad de comprobar un saber a través de una respuesta correcta; incluso en la modalidad de escritura de ensayos (como las pruebas de escritura), que apelan a cierto campo de respuestas posibles (medidas a través de rejillas de evaluacióx n) a partir de estímulos dados. Dado que se trata de un aparato de escritura, las competencias escritas de los individuos quedan sujetas a la medida de una eficacia espacio-temporal: obtener la mayor cantidad de respuestas correctas o esperables en un tiempo limitado y en un espacio inmóvil y plenamente vigilado. Juegos que terminan siendo decisivos como formas de decir la verdad, cierta verdad, sobre los individuos, sobre su vida pasada y futura, que entra como caso individual en un entramado documental.

De acuerdo con lo anterior, podemos afirmar que las capacidades de crítica y autonomía que virtualmente pueden tener cuerpos y poblaciones en una sociedad quedan sujetas a este poder de escritura. En consecuencia, las potencias de transformación de estas fuerzas quedan sojuzgadas en aparatos de encauzamiento de la conducta. Ahora se ve con más claridad cuál puede ser la relación entre la disociación de fuerzas del cuerpo, operadas por las técnicas disciplinarias, y la consigna ilustrada que disocia el uso público del uso privado de la razón. En los aparatos disciplinarios, como el examen, los individuos funcionan como piezas de una máquina, tal como lo señala Kant con respecto al uso privado de la razón. Estos aparatos están articulados a un dispositivo económico que normaliza las capacidades de crítica y autodeterminación de los individuos por medio de exámenes, diagnósticos, registros, sistemas de vigilancia, sanciones y estímulos. Aunque las preguntas de los examinadores pidan a los sujetos razonar, esas mismas preguntas les ordenan obedecer.

Como decíamos al principio, la crítica está en el corazón de las relaciones entre el gobierno de los otros y el gobierno de sí. En la educación superior colombiana la crítica es justamente objeto del poder de evaluar de quienes nos gobiernan. Se nos interpela, a la manera de una consigna ilustrada, para que ejerzamos nuestro poder de evaluar y decidir, pero el mecanismo mismo de interpelación opera a la vez una domesticación de ese poder. Ahora bien, con ello, las posibilidades de ejercer la crítica, como indocilidad reflexiva, no quedan clausuradas. Estas, por el contrario, de acuerdo con las sugerencias de Foucault, pueden residir en el coraje que tengan los actores educativos para restituir el gobierno de sí frente a los excesos del gobierno de los otros; sostener con firmeza el ejercicio público de la crítica frente a los aparatos de gobierno que pretenden reducirla a su uso privado. En este trabajo espero haber contribuido a ese ejercicio crítico. Queda abierta la pregunta de cómo han operado y qué han significado las manifestaciones de la crítica en los movimientos de resistencia o en las contraconductas locales frente a los mecanismos que pretenden domesticarla.

\section{Referencias}

Castro-Gómez, S. (2016a). Historia de la gubernamentalidad II. Filosofía, cristianismo y sexualidad en Michel Foucault. Bogotá: Siglo del Hombre, Universidad Javeriana, Universidad Santo Tomás.

Castro-Gómez, S. (2016b). La vida como obra de arte: Michel Foucault y la estética de la existencia. En A. Lozano-Vásquez y G. A. Meléndez (Comps). Convertir la vida en arte: una introducción histórica a la filosofía como forma de vida (pp. 331-350). Bogotá: Universidad Nacional de Colombia.

Congreso de Colombia. (2009). Ley 1324 de 2009. Por la cual se fijan parámetros y criterios para organizar el sistema de evaluación de resultados de la calidad de la educación, se dictan normas para el fomento de una cultura de la evaluación, en procura de facilitar la inspección y vigilancia del Estado y se transforma el ICFES. Recuperado de: http://mineducacion.gov.co/1621/ articles-210697_archivo_pdf_ley_1324.pdf.

Deleuze, G. (2006). Postscriptum sobre las sociedades de control. Polis, 13. Disponible en: https://polis.revues. org/5509.

Fairclough, N. (1993). Critical Discourse Analysis and the Marketization of Public Discourse: The Universities. Discourse and Society, 4, 133-168.

Foucault, M. (1992). El orden del discurso. Lección inaugural en el Collège de France. Buenos Aires: Tusquets.

Foucault, M. (2007). Nacimiento de la biopolítica. Curso en el Collège de France (1978-1979). Buenos Aires: Fondo de Cultura Económica.

Foucault, M. (2009). Vigilar y castigar. Nacimiento de la prisión. México: Siglo XXI. 
Foucault, M. (2011a). El gobierno de sí y de los otros. Curso del Collège de France (1982-1983). Madrid: Akal.

Foucault, M. (2011b). Seguridad, territorio, población. Curso del Collège de France (1977-1978). Buenos Aires: Fondo de Cultura Económica.

Foucault, M. (2013a). ¿Qué es la crítica? (Crítica y Aufklärung). En: Sobre la Ilustración (pp. 3-52). Madrid: Tecnos.

Foucault, M. (2013b). ¿Qué es la Ilustración? En: Sobre la Ilustración (pp. 71-97). Madrid: Tecnos.

Lazzarato, M. (2013). La fábrica del hombre endeudado. Ensayo sobre la condición neoliberal. Buenos Aires: Amorrortu.

Ministerio de Educación Nacional (MEN). (2009a). Decreto 3963 de 2009. Por el cual se reglamenta el Examen de Estado de Calidad de la Educación Superior. Recuperado de: http://www.mineducacion.gov.co/ normatividad/1753/articles-205955_archivo_pdf_ decreto3963.PDF
Ministerio de Educación Nacional (MEN). (2009b). Boletín informativo No. 13. Educación superior. Recuperado de: http://www.mineducacion.gov.co/1621/articles-92779_archivo_pdf_Boletin13.pdf

Ministerio de Educación Nacional (MEN) . (2011). Propuesta de lineamientos para la formación por competencias en educación superior. Recuperado de: http://www. mineducacion.gov.co/cvn/1665/w3-article-264156. html.

Santos, D. (2013). Los discursos de la exclusión social y el análisis crítico del discurso como acción política: reflexiones a partir del análisis textual de un documento de política educativa universitaria. Discurso y Sociedad, 7(4), 741-763. Recuperado de: http://www.dissoc. org/ediciones/v07n04/DS7(4)Santos.pdf

Soler, S. (2011). Análisis crítico del discurso de documentos de política pública en educación. Forma y Función, 24(1), 75-105. Disponible en: http://www.redalyc.org/ articulo.oa?id=21922416005 